\section{JTI}

JOURNAL OF

TRAUMA AND INJURY

\title{
Sternal Fracture Fixation with a Steel Wire: The New "Timala" Technique
}

\author{
Rabindra Bhakta Timala, M.S. ${ }^{1,2}$, Nirmal Panthee, Ph.D. ${ }^{2}$ \\ ${ }^{1}$ Department of Cardiothoracic and Vascular Surgery, B \& B Hospital, Lalitpur, Nepal \\ ${ }^{2}$ Department of Cardiac Surgery, Shahid Gangalal National Heart Center, Kathmandu, \\ Nepal
}

Received: January 27, 2021

Revised: May 20, 2021

Accepted: May 31, 2021

\section{Correspondence to}

Rabindra Bhakta Timala, M.S. Department of Cardiothoracic and Vascular Surgery, B \& B Hospital, Gwarko, Lalitpur, Nepal

Tel: +977-9851100923

E-mail: rabindratimala@gmail.com ORCID: https://orcid.org/0000-00024907-4743
Purpose: Traumatic sternal fractures are rare but quite disabling injuries. Timely fixation of sternal fractures reduces pain and prevents respiratory complications. However, the fixation technique should be simple, effective, and readily available in local circumstances.

Methods: From January 2014 to March 2020, seven patients with sternal fracture/ dislocation underwent steel wire fixation with the new "Timala" technique. In this technique, adjacent ribs are anchored with two steel wires to form an " $\mathrm{X}$ " in front of the fractured segment of the sternum. Patients were followed up clinically and radiologically. Results: Six of the patients were men and one was a female. Five of them had injuries due to falls and two were injured in road traffic accidents. Their age ranged from 18 years to 76 years, with a median age of 41 years. All seven patients experienced immediate recovery from pain and showed evidence of fracture healing on postoperative chest $\mathrm{X}$-rays and clinical examinations.

Conclusions: Anchoring ribs to fix the sternum with steel wire is a safe, effective, easily available, and reproducible method to fix sternal fractures or dislocations.

Keywords: Fracture; Sternum; Bone wires

\section{INTRODUCTION}

Sternal fractures are associated with blunt anterior chest trauma and are present in $3 \%$ to $8 \%$ of all blunt trauma patients [1-4]. Most sternal fractures are treated conservatively $[1,3,5]$. Surgical fixation is indicated for fracture instability, displacement, pain, and non-union, or for cosmetic purposes. An isolated sternal fracture is often a relatively benign injury $[2,3,6]$. Mortality and morbidity are mostly determined by associated injuries, with rates ranging from $4 \%$ to $45 \%[2,3,5]$.

This is an Open Access article distributed under the terms of the Creative Commons Attribution Non-Commercial License (http://creativecommons.org/licenses/by-nc/4.0/) which permits unrestricted noncommercial use, distribution, and reproduction in any medium, provided the original work is properly cited. 
Surgical plating provides more stability and better restoration of anterior chest wall function than wiring, including improved bone healing and a lower incidence of complications and non-union [1,2,6-9]. Although titanium plates with locking screws seem to be popular in developed countries $[7,10]$, in underdeveloped countries they are often unavailable or surgeons are not properly trained to perform fixation with plates and screws. This results in patients not being treated properly. Without proper treatment, patients often have to endure dyspnea, long-term pain, malunion, cosmetic deformities, and prolonged hospitalization. There is a need for a simple, safe, and effective method for sternal stabilization. Herein, we describe our technique of sternal fracture fixation with steel wire that is simple and economical, yet effective and reproducible.

\section{METHODS}

After obtaining ethical approval from the Institutional Review Board of B \& B Hospital, we conducted a retrospective review of patients who had undergone sternal fracture fixation over the last 6 years. From January 2014 to March 2020, seven patients with sternal fractures due to blunt trauma underwent sternal fracture fixation with this new technique of wire fixation. The operative criteria were debilitating pain, displacement of the fracture, and malunion.

Baseline blood and metabolic investigations, electrocardiography (ECG), cardiac enzyme (CPK-MB), and echocardiography were done upon arrival in the emergency room to rule out associated cardiac injuries. ECG and CPK-MB were repeated after 12 hours. All patients underwent chest X-ray and computed tomography (CT) scans of the chest with 3-dimensional reconstruction before surgery. The fixation device consisted of no. 5 stainless steel wire, which is usually used for sternal closure after sternotomy in cardiac surgery.

Surgery was done within 1 week of trauma in all but one patient, who underwent surgery after 3 weeks due to malunion. Surgery was performed under general anesthesia with single-lumen endotracheal intubation. Patients were laid supine with a roll under the shoulder to slightly hyperextend the anterior chest wall. In the initial series, a longitudinal skin incision over the midline of the sternum was made. However, in the last two cases, a transverse skin incision just over the fracture site was made. The sternum, adjacent costal cartilage, and ribs were exposed. If needed, fractures were reduced with metal retractors and perioste-
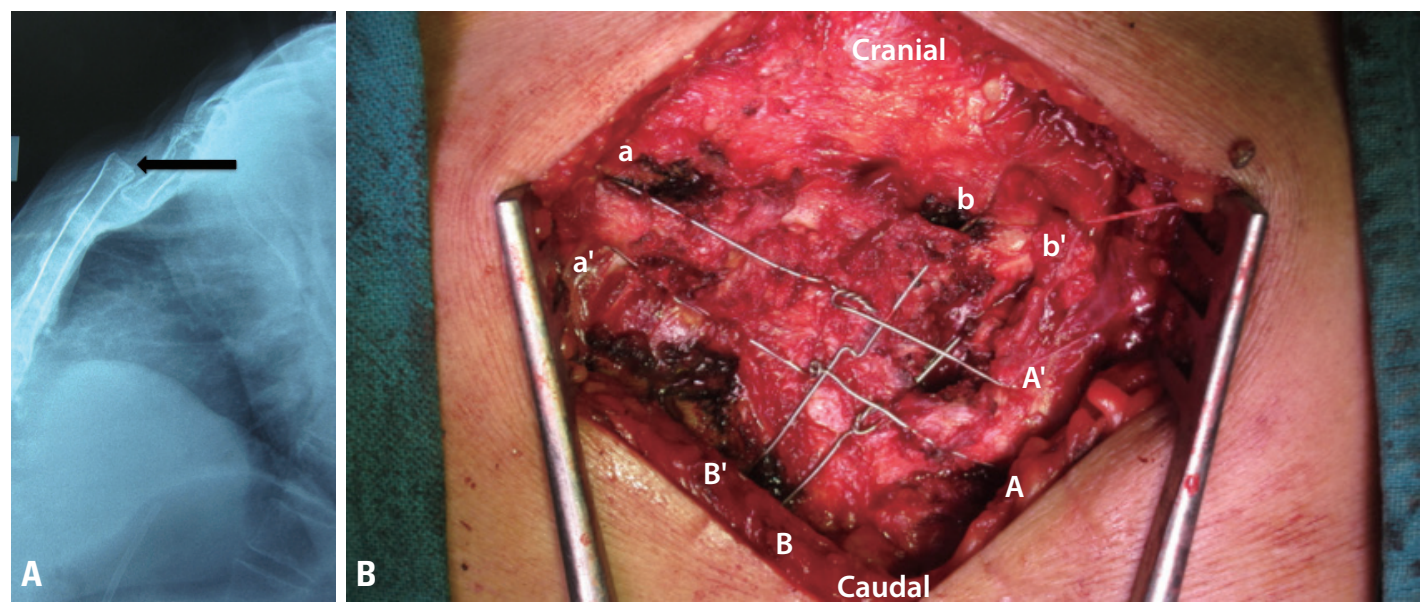

Fig. 1. (A) Chest X-ray (lateral view) showing manubriosternal joint dislocation (arrow). This type of fracture requires reduction using metal retractors and/or periosteal elevators before fixation using our technique. (B) Representative picture at the completion of surgery. One steel wire is passed through the body of the lower rib (from A to A'). The same wire is then passed through the body of the upper rib (from a to $a^{\prime}$ ) on the contralateral side; and tied in front of the sternum. Next, a steel wire is passed through the body of the lower rib (from B to B'); this same wire is then passed through the body of the upper rib (from $b$ to $b^{\prime}$ ) on the contralateral side; and tied in front of the sternum. The final appearance is that the two steel wires form an " $X$ " in front of the fractured segment of the sternum. 


\section{JTI}

al elevators (Fig. 1A). An important caveat was to expose the adjacent ribs, 1 above and 1 below the fracture level on each side. Where it was not possible to expose the upper ribs (the first ribs in case of sterno-manubrial dislocation), the ribs at the same level as the fracture site (the second ribs in that instance) were exposed. A no. 5 stainless steel wire was taken, and the needle was passed through the rib (up or down from the fracture level) in transverse manner. We traversed the body of the rib with wire not encircling the rib. Technically, this is somewhat tricky given the shape of the needle of the sternal wire. Care was taken not to take a bite through the cartilaginous portion, as this was found to be brittle and fragile, by not holding the wire when force was applied to tighten, compared to the bony part of the rib. The same needle was then used to take a bite on the rib on the other side. If the previous bite was on the lower level, the next bite should be on the upper level on the other side and vice-versa. Another wire was used to take a bite on a different rib level on either side. The free ends of the wire were tied in front of the sternum. The amount of force needed to tie the wires was judged based on the visual orientation and approximation of the fractured segments during wire tightening, so that sternal depression would not occur at a later stage. Therefore, the wires ultimately formed an " $\mathrm{X}$ " in front of the sternum (Fig. 1B). Muscles, subcutaneous tissue, and skin were closed in the conventional manner. Drains were not kept. Intraoperative imaging was not done. Patients were transferred to the postoperative ward or intensive care unit depending upon their associated injuries. Routine postoperative chest X-rays were done on the same day and the next morning. The usual postoperative care was given.

After discharge from the hospital, patients were followed up at 1,3 , and 12 months after surgery and yearly thereafter. Chest X-ray postero-anterior and lateral views were done to assess healing in all patients, and a CT scan was done in one patient (Fig. 2).

Table 1. Clinical characteristics of the patients

\begin{tabular}{|lc|}
\hline Variable & Value $(\mathbf{n}=\mathbf{7})$ \\
\hline Sex (male) & $6(86.0)$ \\
Age (years) & $41(18-76)$ \\
Associated injuries & \\
Spinal injury & $1(14.3)$ \\
Spinal injury+limb injury & $1(14.3)$ \\
Spinal injury+rib fracture & $1(14.3)$ \\
Rib fracture & $1(14.3)$ \\
Rib fracture+limb fracture & $1(14.3)$ \\
Sternal fracture types and location & \\
Upper sternal body & $4(57.0)$ \\
Manubriosternal joint dislocation & $2(28.6)$ \\
Lower sternal body & $1(14.3)$ \\
\hline
\end{tabular}

Values are presented as number (\%) or median (interquartile range).
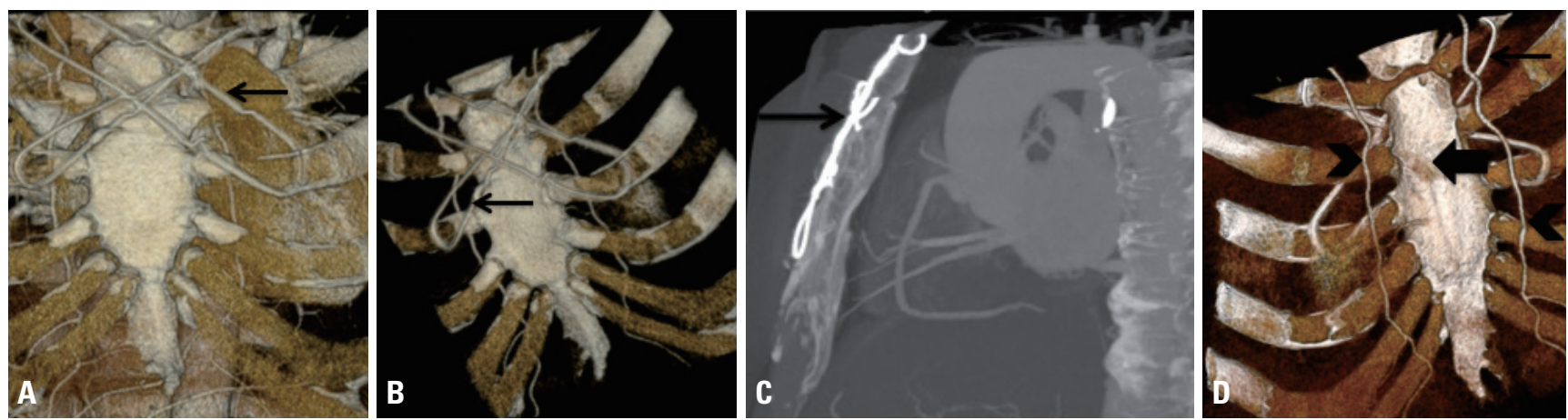

Fig. 2. Postoperative computed tomography (CT) scan of a 48-year-old male patient who had undergone sternal fracture fixation using our technique 3 years ago. A coronary CT angiogram was done to rule out coronary artery disease, at which time the previously fixed sternal fracture was evaluated using 3D reconstruction. (A) True anteroposterior view showing the sternal wires forming an " $X$ " in front of the sternum. (B) Anteroposterior view with some degree of oblique orientation showing a perfectly aligned sternum. (C) True lateral view showing the healed sternum; it should be noted that the wires are in front of the sternum. (D) Postero-anterior view showing a healed fracture at the level of the third rib. It should be noted that the wires are not behind the sternum (arrow: steel wire, thick arrow: healed fracture, arrowheads: bilateral internal mammary arteries). 


\section{RESULTS}

The clinical characteristics of the patients are presented in Table 1 . The vast majority (86\%) of the patients were men. The median age of the patients was 41 years (range 18-76 years). Almost two-thirds $(\mathrm{n}=5)$ had sustained fall injuries, and the remaining $29 \%(n=2)$ had been in road traffic accidents. An isolated sternal fracture or manubriosternal dislocation was present in only two patients (29\%). Spinal injury $(43 \%, n=3)$ and rib fractures $(43 \%$, $\mathrm{n}=3$ ), either alone or in combination, were the commonest associated injuries (Table 1).

Slightly more than half of the patients $(57 \%, n=4)$ had transverse upper sternal body fractures, two had manubriosternal joint dislocations (one of whom had malunion and presented 3 weeks after trauma), and the remaining patient had a lower sternal body fracture (Table 1).

All seven patients had an uneventful recovery. Pain was relieved immediately. Patients' postoperative hospital course was mainly determined by their associated injuries, rather than by sternal fracture fixation. Complications such as wound infection, hematoma, pneumothorax, continuing pain, re-dislocation of fracture segments, malunion, or non-union were not observed. None of the patients died during hospitalization and surgery.

The total follow-up was 23 patient-years. During follow-up, all patients healed well and had a well-aligned sternum. Sternal stability was achieved in all patients. Clinical examination findings and chest X-rays (postero-anterior and lateral views) were normal. None of the seven patients required reoperation for non-union or malunion.

\section{DISCUSSION}

Sternal fractures and dislocation are rare injuries [13,11,12]. A recent meta-analysis by Klei et al. [12] found only 354 patients over 56 years and 3 months, although they included only 191 patients in their analysis.

Five out of seven patients in our study sustained a fall injury leading to sternal fracture, in contrast to studies from developed countries, where the cause is usually a seat belt injury associated with motor vehicle injury $[13,14]$.
The role of cardiac biomarkers in sternal injuries has been debated. Normal troponin I and T after 4-6 hours following blunt chest injury indicates that hemodynamically stable patients can be discharged home safely [15]. A group of researchers from London showed that CPK-MB measurements were non-contributory following sternal fractures [16]. They further argued that isolated sternal fractures, in the absence of clinical evidence of cardiac or respiratory complications, and with a normal electrocardiogram and postero-anterior chest radiograph, require no further investigation [16]. However, another group from the University of Tennessee showed that CPK-MB is the most reliable indicator of myocardial injury [17]. We believe that measuring $\mathrm{CPK}-\mathrm{MB}$ in patients with sternal fractures is a relatively easy step to obtain a general impression of cardiac status before proceeding to surgery, and we therefore routinely measure CPK-MB in all patients.

Chest CT helped us to establish the fragment position, as well as to look for other injuries in regions such as the aorta, heart, lungs, or spine. During follow-up, a chest $\mathrm{X}$-ray lateral view was sufficient to assess the position of sternal fragments and healing; therefore, we did not order CT scans during follow-up due to economic reasons except in one patient. This patient was a 48-year-old male who had undergone sternal fixation 3 years previously. This time, his clinical presentation was suspicious for coronary artery disease and we performed a coronary CT angiogram. His CT scan showed favorable healing of the fractured segment (Fig. 2) and his coronary arteries were normal.

Traditionally, sternal fractures have been fixed mostly by plates and screws (with $99 \%$ of cases being treated by plates), with good results in terms of pain relief and fracture healing [12]. Plating provides more stability and better chest wall function, as well as a decreased chance of non-union and better bone healing than is possible with wires $[1,2,4,8]$. However, many surgeons are reluctant to perform sternal osteosynthesis for various reasons, such as difficulty in preoperative planning, severe injuries to mediastinal organs, and lack of adequate training in this procedure. Drilling too deep may cause life-threatening injuries to mediastinal organs. In their meta-analysis, Klei et al. [12] found that $10 \%$ of patients $(15 / 145)$ required 
removal of the osteosynthesis. Harston and Roberts [4] found that $19 \%$ of surgically treated patients suffered from complications, including osteosynthesis removal. Ciriaco et al. [18] described having to remove the titanium cervical plate used for sternal fracture fixation in one of seven patients due to severe chest discomfort, and the patients' symptoms improved after removal.

We changed our strategy from longitudinal incision to transverse incision in the last two cases. Unlike with titanium plates, which always require a longitudinal incision, our technique can utilize either a longitudinal or a transverse incision. We believe that a transverse incision offers a cosmetic advantage, as an incision along the Langer line would create a less noticeable scar than one going across it. Furthermore, because of the transverse nature of sternal fractures, it is not necessary to expose the sternum longitudinally, and our area of interest (i.e., rib edges) is easier to access with a transverse incision than with a longitudinal incision.

The classical technique of wire fixation involves taking bites or drilling holes through the fractured sternal plates and joining them. Extensive dissection, dividing the intercostal muscles, is required to mobilize the fractured segments sufficiently to be able to pass a steel suture through, risking injury to the internal mammary artery. The pericardium and heart might also be injured in this process. Therefore, this procedure is not simple, although it may seem so. A group of researchers from the University of Minnesota described their series of 12 patients with traumatic sternal fractures without internal organ injury using a drill, wires, and Steinmann pin to treat the sternal fractures, which may be overkill [19]. In their series, one of the patients had protrusion of the Steinmann pin under the skin, requiring pin removal [19].

This study is a case series of sternal fracture fixation procedures performed by a single surgeon at a hospital in Nepal. The technique described by the authors does not need extensive dissection of muscles or bone. Stainless steel wires do not need to pass through the posterior periosteum of the sternum or ribs, which simplifies the procedure and makes it safe. Therefore, this procedure is unlikely to injure any vital organs or the internal mammary artery or cause pneumothorax, and does not require any sophisticated equipment. Stainless steel wires are gener- ally available in any part of the world and cost much less than titanium plates. A titanium plate with a screw would cost roughly 200 USD in Nepal, and the sternal steel wire would cost only 2 USD. Therefore, there is a 100 -fold price difference in the material only, ignoring other factors. Our technique is significantly more economical than the plating technique. In addition, our technique avoids concerns regarding the possibility of injuring underlying structures, whereas that is a relevant concern with screws in the plating technique. Nonetheless, it would have been preferable to compare our technique with the plating technique at a large-volume center that practices titanium plating for sternal fractures. Moreover, wires are less likely than plates to cause patient discomfort and skin-related problems.

Because of the complexity of other treatment methods, sternal fractures are usually not treated surgically; instead, conservative observation has been recommended $[20,21]$. More than $95 \%$ of patients with a fractured sternum are treated conservatively [19]. Initial conservative management might lead to a hunched posture, restricted neck extension, and kyphosis [19]. Simple and effective treatments like the one we describe here can overcome this hurdle, especially in developing countries. Fracture non-union is a rare entity in sternal fractures, with an incidence of $<1 \%[22,23]$. Therefore, a simple approximation of the fractured fragments, as we describe in our technique, is good enough for most patients. Given the lack of standardized treatment guidelines and randomized controlled trials for sternal fractures and dislocation, our technique adds a new but simple treatment strategy for the management of sternal fractures.

\section{Limitations}

To our knowledge, this study is the first of its kind to be reported from Nepal. As a retrospective case series, it has potential limitations. The sample size was small, but this reflects the rarity of the condition. Pain relief was not quantified. Follow-up ranged from 10 months to 7 years, meaning that delayed sternal non-healing could not be assessed in all patients. Chronic non-union cases with or without bone grafts were not treated with this method, so generalizability to this particular group of patients is not possible. This technique of sternal fixation using wires can 
be used for any general indication of sternal fracture fixation, such as fracture fragment instability, displacement, pain, or non-union, as well as for cosmetic purposes. If one or two costochondral fractures are present, our technique can still be used, though adjacent non-fractured ribs should be used for anchoring the wire. However, the presence of a series of costochondral fractures, which is very unlikely, might contraindicate this procedure.

\section{CONCLUSION}

Sternal fracture fixation using ribs as anchoring points for the wire is a useful technique with good and reproducible results. Randomized controlled trials comparing plating with this new "Timala" technique should be done at high-volume centers in order to further clarify its usefulness.

\section{REFERENCES}

1. Zhao Y, Yang Y, Gao Z, Wu W, He W, Zhao T. Treatment of traumatic sternal fractures with titanium plate internal fixation: a retrospective study. J Cardiothorac Surg 2017;12:22.

2. Krinner S, Grupp S, Oppel P, Langenbach A, Hennig FF, SchulzDrost S. Do low profile implants provide reliable stability in fixing the sternal fractures as a "fourth vertebral column" in sternovertebral injuries? J Thorac Dis 2017;9:1054-64.

3. Athanassiadi K, Gerazounis M, Moustardas M, Metaxas E. Sternal fractures: retrospective analysis of 100 cases. World J Surg 2002;26:1243-6.

4. Harston A, Roberts C. Fixation of sternal fractures: a systematic review. J Trauma 2011;71:1875-9.

5. Knobloch K, Wagner S, Haasper C, Probst C, Krettek C, Otte D, et al. Sternal fractures occur most often in old cars to seat-belted drivers without any airbag often with concomitant spinal injuries: clinical findings and technical collision variables among 42,055 crash victims. Ann Thorac Surg 2006;82:444-50.

6. Byun CS, Park IH, Hwang WJ, Lee Y, Cho HM. Analysis of sternal fixation results according to plate type in sternal fracture. Korean J Thorac Cardiovasc Surg 2016;49:361-5.

7. Divisi D, Di Leonardo G, Crisci R. Surgical management of traumatic isolated sternal fracture and manubriosternal dislo- cation. J Trauma Acute Care Surg 2013;75:824-9.

8. Schulz-Drost S, Oppel P, Grupp S, Schmitt S, Carbon RT, Mauerer A, et al. Surgical fixation of sternal fractures: preoperative planning and a safe surgical technique using locked titanium plates and depth limited drilling. J Vis Exp 2015;(95):e52124.

9. Nazerali RS, Hinchcliff K, Wong MS. Rigid fixation for the prevention and treatment of sternal complications. Ann Plast Surg 2014;72(Suppl 1):S27-30.

10. Gandy KL, Moulton MJ. Sternal plating to prevent malunion of transverse sternotomy in lung transplantation. Ann Thorac Surg 2008;86:1384-5.

11. Kälicke T, Frangen TM, Müller EJ, Muhr G, Hopf F. Traumatic manubriosternal dislocation. Arch Orthop Trauma Surg 2006;126:411-6.

12. Klei DS, de Jong MB, Öner FC, Leenen LPH, van Wessem KJP. Current treatment and outcomes of traumatic sternal fractures-a systematic review. Int Orthop 2019;43:1455-64.

13. Trinca GW, Dooley BJ. The effects of mandatory seat belt wearing on the mortality and pattern of injury of car occupants involved in motor vehicle crashes in Victoria. Med J Aust 1975;1:675-8.

14. Brookes JG, Dunn RJ, Rogers IR. Sternal fractures: a retrospective analysis of 272 cases. J Trauma 1993;35:46-54.

15. Collins JN, Cole FJ, Weireter LJ, Riblet JL, Britt LD. The usefulness of serum troponin levels in evaluating cardiac injury. Am Surg 2001;67:821-5; discussion 825-6.

16. Heyes FL, Vincent R. Sternal fracture: what investigations are indicated? Injury 1993;24:113-5.

17. Fabian TC, Mangiante EC, Patterson CR, Payne LW, Isaacson ML. Myocardial contusion in blunt trauma: clinical characteristics, means of diagnosis, and implications for patient management. J Trauma 1988;28:50-7.

18. Ciriaco P, Casiraghi M, Negri G, Gioia G, Carretta A, Melloni G, et al. Early surgical repair of isolated traumatic sternal fractures using a cervical plate system. J Trauma 2009;66:462-4.

19. Molina JE. Evaluation and operative technique to repair isolated sternal fractures. J Thorac Cardiovasc Surg 2005;130:445-8.

20. Potaris K, Gakidis J, Mihos P, Voutsinas V, Deligeorgis A, Petsinis V. Management of sternal fractures: 239 cases. Asian Cardiovasc Thorac Ann 2002;10:145-9.

21. Sadaba JR, Oswal D, Munsch CM. Management of isolated sternal fractures: determining the risk of blunt cardiac injury. Ann R Coll Surg Engl 2000;82:162-6.

22. Queitsch C, Kienast B, Voigt C, Gille J, Jürgens C, Schulz AP. 


\section{JTI}

Journal of Trauma and Injury Volume 34, Number 3, September 2021

Treatment of posttraumatic sternal non-union with a locked sternum-osteosynthesis plate (TiFix). Injury 2011;42:44-6.

23. Wu LC, Renucci JD, Song DH. Sternal nonunion: a review of current treatments and a new method of rigid fixation. Ann Plast Surg 2005;54:55-8. 